\title{
Mini External Fixator for Management of Fracture Metacarpals and Phalanges of the Hand
}

\author{
Ahmed Al Saeed Abd Allah*, Khaled IdreesAbd Al Rahman, \\ Mohammed El Sadek Attia, Ahmed Mohammed Abdel Wahab \\ Department of Orthopedic Surgery, Faculty of Medicine, Zagazig University, Egypt \\ *Corresponding author: Ahmed Al Saeed Abd Allah, Mobile: (+20) 10-9300862, E-mail: draaasa@gmail.com
}

\begin{abstract}
Background: Fractures of metacarpals and phalanges are the most common fractures of the upper extremity. Objective: The aim of this study was to assess the functional outcomes of management of metacarpal and phalangeal fractures by mini external fixator.

Patients and Methods: This prospective study included a total of 12 patients with fractures of metacarpals and phalanges, attending at Orthopedics and Traumatology Departments, Zagazig and Helwan University Hospitals. This study was conducted between August 2018 and July 2019 and was done to assess the functional outcomes of metacarpal and phalangeal fractures fixed by mini external fixator.

Results: In this study there was good union time among studied group with average $8.33 \pm 2.1$ weeks. One case $(8.3 \%)$ had pin tract infection and one case $(8.3 \%)$ had delayed union $>12$ weeks, malunion founded in 2 cases $(16.7 \%)$ and stiffness founded in 3 cases $(25 \%)$. At the end of the follow up period, two patients $(16.7 \%)$ sustained malunion deformities, one patient $(8.35 \%)$ had rotational deformity and in another patient $(8.35 \%)$ had angular malunion. Regarding active movement score $75 \%$ were acceptable results (Excellent and Good) and 25\% were not acceptable results (Fair and Poor). There was significant association between acceptability of outcome and deformity, as $66.7 \%$ of not accepted result had deformity while no one from accepted outcome had deformity.

Conclusion: Use of mini external fixator is an adequate procedure for unstable phalangeal and metacarpal fractures. In cases of open fractures, the mini external fixator allows wound inspection and care and avoidance of hardware application at fracture site.
\end{abstract}

Keywords: Metacarpal and Phalangeal Fractures, Mini External Fixator.

\section{INTRODUCTION}

The human hand is the most part of the body susceptible to a wide range of injuries especially in manufacturing, agricultural field and adding to this increased road traffic accidents resulting in high incidence of phalangeal and metacarpal fractures particularly mutilating hand injuries, open fractures, comminuted fractures and intra articular fractures ${ }^{(\mathbf{1})}$.

Metacarpal and phalangeal fractures are the most common fractures of the upper extremity that account for $10 \%$ of total body fractures. The outer parts of the hand are the most commonly parts to be injured. The incidence of metacarpal and phalangeal fractures is most common in males and peaks at the age of 10-40 years when the athletic injury and industrial exposure is the greatest ${ }^{(2)}$.

The bad thing among these fractures that the metacarpal and phalangeal fractures are often neglected or regarded as trivial injuries ${ }^{(3)}$. Proximal phalanges of the fingers are fractured more frequently than the middle or even distal phalanges. Deformity leads to displacement is typical to occur when the proximal phalanges are fractured ${ }^{(4)}$.

Treatment with closed procedure has led to bad outcomes as the malunion, stiffness and loss of soft tissues or skin over the injured part. Type of surgery for fixation depends on several factors that include fracture geometry, location, deformity and if the wound is closed or opened plus the stability of the fracture ${ }^{(5)}$.

Non-operative treatments like elevation, rest, splinting, cast with or without traction, open reduction and internal fixation by k-wire, plates, screws etc., leads to more damage of the already injured soft tissues, stiffness of the joints and delay in rehabilitation. External fixation allows fracture reduction to normal bony length via a rigid external support ${ }^{\left({ }^{(}\right)}$.

The aim of this study was to assess the functional outcomes of management of metacarpal and phalangeal fractures by mini external fixator.

\section{PATIENTS AND METHODS}

This prospective study included a total of 12 patients with fractures of metacarpals and phalanges, attending at Orthopedics and Traumatology Departments, Zagazig and Helwan University Hospitals. This study was conducted between August 2018 and July 2019.

The mini-external fixator was used to fix the fractures within one week and patients were followed up for 12-36 months with an average period of 24 weeks.

Inclusion criteria: Patients aged between 17 and 50 years with unstable fractures of metacarpals or 
phalangeal intra articular and extra-articular fractures and fractures were open fractures, comminuted fractures or multiple fractures.

Exclusion criteria: Patients with severely crushed hand injuries or fractures associated with tendon injuries or neurovascular injuries.

The patients were assessed clinically by full history taking, general and local examination and radiological evaluation of the fractures.

\section{Surgical Technique:}

The operation was carried out on 7 patients $(58.3 \%)$ under general anesthesia while the remaining 5 patients $(41.7 \%)$ were regionally anesthetized.

Percutaneous pinning one Kirschner wire was inserted proximally and distally to the fracture. Using the Double Drill Sleeve the Kirschner wires were inserted until their tips are anchored in the far cortical bone. Making sure that the Kirschner wires are aligned in one plane.

The Kirschner wires were inserted into the metacarpals and phalanges using a dorso-radial or a dorso-ulnar approach.

To place the Kirschner wires in the proximal part of the first phalanges, the extensor hood was incised in the direction of the movement of the tendon.

The Kirschner wires were introduced laterally into the metacarpals I, II.

Holding Clamp was slided on each Kirschner wire by its slotted opening and slightly tighten the screw nut by hand.

In order to determine the entry points of the secondary Kirschner wires, Kirschner wire was introduced into the vacant openings of the clamps, The skin was marked and incised and also the subjacent soft tissue. The Kirschner wire used for marking then removed. Slide a Connecting Rod through both holding clamps and tighten it to one of them using the Socket.

The fracture was reduced, and the reduction fixed by slightly tightening the screw nut for the Kirschner wires. Tightening the nut was done to fix the reduction, making sure the vacant opening is still wide enough so that the parallel secondary Kirschner wire introduced later. Securely tightened the screw nuts for the connecting rod.

Retracting the soft tissue along the incision with small bone hooks and inserting the secondary Kirschner wires through the vacant openings of the holding clamps were done while cooling the wire and the clamps.

The screw nuts for the Kirschner wires were tightened and simultaneously hold the connecting rod with the Clamp Holder.

\section{Methods of Evaluation:}

A follow up protocol included clinical evaluation for the immensity of pain, stability of the fractures and the range of the distal and proximal joints. Complications were also identified and managed. This was done in the first two weeks then monthly for four months postoperatively.

Routine plain radiographs were done before the discharge of the patients and every two weeks for three months. Evaluating the healing of fracture, signs of loosening of the wires.

Assessment of the results was based upon the American Society for Surgery of the Hand (ASSH) Total Active Movements score (119). Functional assessment was done based on total active range of movements in degrees of each injured finger separately.

\section{Data management:}

Data collected throughout history, basic clinical examination, laboratory investigations and outcome measures coded, entered and analyzed using Microsoft Excel software. Data were then imported into Statistical Package for the Social Sciences (SPSS version 20.0) (Statistical Package for the Social Sciences) software for analysis. According to the type of data qualitative represent as number and percentage, quantitative continues group represent by mean $\pm \mathrm{SD}$, the following tests were used to test differences for significance difference and association of qualitative variable by Chi square test $\left(\mathrm{X}^{2}\right)$. Differences between quantitative independent groups by $\mathrm{t}$ test. $\mathrm{P}$ value was set at $<0.05$ for significant results $\&<0.001$ for high significant result.

\section{Ethical Considerations:}

Institutional Review Board (IRB) of the Faculty of Medicine, Zagazig University approved the study protocol (No.4381). The work has been carried out in accordance with The Code of Ethics of the World Medical Association (Declaration of Helsinki) for studies involving humans. An informed consent was obtained from all participants of this study and they were told about the aim of the study and were informed that the data would be used for scientific purposes only.

\section{Statistical analysis}

Data collected throughout history, basic clinical examination, laboratory investigations and outcome measures coded, entered and analyzed using Microsoft Excel software.

Data were then imported into Statistical Package for the Social Sciences (SPSS version 20.0) (Statistical Package for the Social Sciences) software for analysis. According to the type of data qualitative represent as number and percentage, quantitative continues group represent by mean $\pm \mathrm{SD}$, the following tests were used to test differences for significance;.. Differences between quantitative paired groups by paired $t, P$ value was set at $<0.05$ for significant results $\&<0.001$ for high significant result. 


\section{RESULTS}

Union time was distributed as $8.33 \pm 2.1$ week with minimum 6 weeks and maximum 13 weeks (one delayed case $>12$ weeks) (Table 1).

The only one case $(8.3 \%)$ had pin tract infection and also one case (8.3\%) had delayed union $>12$ weeks, mal union founded in 2 cases $(16.7 \%)$ and stiffness founded in 3 cases (25\%) (Table 2).

At the end of the follow up period, two patients $(16.7 \%)$ sustained malunion deformities one patient $(8.35 \%)$ had rotational deformity and in another patient $(8.35 \%)$ had angular malunion (Table 3).
According to the system adopted by the American Society for Surgery of the Hand (ASSH) Total Active Movements score, there were 6 patients $(50.0 \%)$ who ended up with excellent results, three patients $(25.0 \%)$ with good results, two patients $(16.7 \%)$ with fair results, and one patient $(8.3 \%)$ with poor results, and overall we had $75 \%$ with acceptable result (Excellent and Good) and 25\% with not acceptable result (Fair and Poor) (Table 4).

There was significant association between Acceptability of outcome and deformity, as $66.7 \%$ of not accepted result had deformity while no one from accepted outcome had deformity (Table 5).

Table (1): Distribution of cases according to union time

\begin{tabular}{|l|l|}
\hline & Union time (weeks) \\
\hline Mean \pm SD & $8.33 \pm 2.1$ \\
\hline Median (Range) & $8.0(6-13)$ \\
\hline
\end{tabular}

\section{SD (Standard Deviation)}

Table (2): Distribution of cases according to complication

\begin{tabular}{|l|l|l|l|}
\hline \multicolumn{2}{|c|}{} & $\mathbf{N}$ & $\%$ \\
\hline \multirow{2}{*}{ Infection } & No & 11 & 91.7 \\
\cline { 2 - 4 } & Yes & 1 & 8.3 \\
\hline \multirow{2}{*}{ Delayed union } & No & 11 & 91.7 \\
\cline { 2 - 4 } & Yes & 1 & 8.3 \\
\hline \multirow{2}{*}{ Mal union } & No & 10 & 83.3 \\
\cline { 2 - 4 } & Yes & 2 & 16.7 \\
\hline \multirow{3}{*}{ Stiffness } & No & 9 & 75.0 \\
\cline { 2 - 4 } & Yes & 3 & 25.0 \\
\cline { 2 - 4 } & Total & 12 & 100.0 \\
\hline
\end{tabular}

Table (3): Distribution of cases according to final end results

\begin{tabular}{|l|l|l|}
\hline & Frequency & Percent \\
\hline No deformity & 10 & 83.3 \\
\hline Deformity & 2 & 16.7 \\
\hline Total & 12 & 100.0 \\
\hline
\end{tabular}

Table (4): Distribution of cases according to final end results

\begin{tabular}{||l|l|l|l|}
\hline \multirow{2}{*}{ Accepted } & Excellent & N & \% \\
\cline { 2 - 4 } & Good & 6 & 50.0 \\
\hline \multirow{2}{*}{ Not accepted } & Fair & 3 & 25.0 \\
\cline { 2 - 4 } & Poor & 2 & 16.7 \\
\hline Total & & 1 & 8.3 \\
\hline \hline
\end{tabular}


Table (5): Correlation between outcome and presence of deformity

\begin{tabular}{|c|c|c|c|c|c|c|}
\hline & & \multicolumn{2}{|c|}{ Acceptability of outcome } & \multirow{2}{*}{ Total } & \multirow{2}{*}{$\mathbf{X}^{2}$} & \multirow{2}{*}{$\mathbf{P}$} \\
\hline & & Accepted & Not accepted & & & \\
\hline \multirow{2}{*}{ No deformity } & $\mathrm{N}$ & 9 & 1 & 10 & \multirow{4}{*}{4.3} & \multirow{4}{*}{$0.045^{*}$} \\
\hline & $\%$ & $100.0 \%$ & $33.3 \%$ & $83.3 \%$ & & \\
\hline \multirow{2}{*}{ Deformity } & $\mathrm{N}$ & 0 & 2 & 2 & & \\
\hline & $\%$ & $0.0 \%$ & $66.7 \%$ & $16.7 \%$ & & \\
\hline \multirow{2}{*}{ Total } & $\mathrm{N}$ & 9 & 3 & 12 & & \\
\hline & $\%$ & $100.0 \%$ & $100.0 \%$ & $100.0 \%$ & & \\
\hline
\end{tabular}

\section{DISCUSSION}

The incidence of fracture in this study was more in males $(83.3 \%)$. and this rightly corresponds to the risk of ambulant life led by males. In Yaseen's et al. ${ }^{(7)}$ study 56 patients, 41 were men and 16 were women.

In the present study, $8(66.7 \%)$ fractures were open and $4(33.3 \%)$ fractures were closed. However in the study that reported by Drenth's ${ }^{(8)}$, there were 27 (81.9\%) patients with open fractures and 25 of them with severe soft tissue injuries (from total 33 patients).

Dailiana and Klasen ${ }^{(9)}$ reported $23(38.9 \%)$ patients with open fractures and in Bakki's et al. ${ }^{(10)}$ study, all fractures were open.

In this work, the most common mechanism of injury was direct trauma either by impaction or crushing in 7 patients $(58.3 \%)$ which were most probably due to falling of heavy objects on the hand, or indirect trauma in the form of twisting injury in 5 patients $(41.7 \%)$.

However in the study of Yaseen et al. ${ }^{(7)}$, the most common mechanism of injury was road traffic accident in 36 patients (64.29\%).

In the presented study, union time was distributed as $8.33 \pm 2.1$ with minimum 6 weeks and maximum 13 weeks.

This was nearly the same as the studies of Drenth and Klasen ${ }^{(8)}$, Dailiana et al. $^{\left({ }^{(9)}\right.}$ and Bakki et $\boldsymbol{a l}^{\left({ }^{(10)}\right.}$ in which union ranged between 3 and 12 weeks.

In this study, one case $(8.3 \%)$ had pin tract infection and also one case had delayed union $>12$ weeks, mal union founded in 2 cases $(16.7 \%)$ and stiffness founded in 3 cases $(25 \%)$.

In Drenth and Klasen ${ }^{(8)}$ study, complication rate was $27.8 \%$ in his mixed study in six patients; one of the pins became loose. Two patients part of the device interfered with the soft tissues of the adjacent fingers, in another patient the device restricted movement of the adjacent fingers and the fracture became displaced. Only in one patient the fracture became displaced due to loosening of wires, which required surgical revision.

\section{CONCLUSION}

It could be concluded that use of mini external fixator is an adequate procedure for unstable phalangeal and metacarpal fractures. In cases of open fractures, the mini external fixator allows wound inspection and care and avoidance of hardware application at fracture site. It can be removed easily in outpatient clinic with no need of second surgery for hardware removal. It is easily applied and allows early mobilization of the surrounding joints which prevents stiffness and other complications.

- Conflict of interest: No

- Financial disclosure: No

\section{REFERENCES}

1. Mishra A, Adhikari V, Chalise $P$ et al. (2019): Jess Fixator for Metacarpal and Phalangeal Fracture. Nepal Medical College Journal, 21(1): 48-52.

2. Bakki S, Yennapu N, Chollangi A et al. (2017): Stabilization of phalangeal and metacarpal fractures with JESS fixation. J. Evid. Based Med. Healthc., 4(12): 661668.

3. Watson-Jones R, Barton N (2009): Fractures and Joint Injuries of the hand. In: Wilson JN, editor. Watson-Jones fractures and joint Injuries. 7th ed. Noida: Elsevier Publishers; Pp. 650-95.

4. Gaspar M, Gandhi S, Poggetti A et al. (2018). Dual Antegrade Intramedullary Headless Screw Fixation for Treatment of Unstable Proximal Phalanx Fractures. The Journal of Hand Surgery, European, 41(7):688-694.

5. Barton N (1989): Internal fixation of the hand. J Hand Surg Br., 14: 139-42.

6. Behrens F (1989): General theory and principles of external fixation. Clin Orthopaedics, 241: 15-23.

7. Yaseen G, Qureshi K, Hussain A et al. (2016): Results of mini externalfixator in metacarpal and phalangeal fractures. PAFMJ., 66(5): 715-19.

8. Drenth D, Klasen H (1998): External fixation for phalangeal and metacarpal fractures. J Bone Joint Surg Br., 80(2): 228-30.

9. Dailiana Z, Agrostakis D, Varitimidis S et al. (2009): Use of a mini external fixator for the treatment of hand fractures. Journal of Hand Surgery, 34(6): 630-6.

10. Bakki S, Yennapu N, Chollangi A et al. (2017): Stabilisation of phalangeal and metacarpal fractures with JESS fixation. J Evid Based Med Healthc., 4(12): 661668. 